\title{
Use of liquid chromatography-mass spectrometry and a chemical cleavage reaction for the structure elucidation of a new sildenafil analogue detected as an adulterant in an herbal dietary supplement
}

\author{
John C. Reepmeyer*, Jeffrey T. Woodruff \\ US Food and Drug Administration, Division of Pharmaceutical Analysis, St. Louis, MO 63101, USA \\ Received 9 February 2007; received in revised form 7 April 2007; accepted 11 April 2007 \\ Available online 18 April 2007
}

\begin{abstract}
An herbal dietary supplement, marketed as a natural product for the enhancement of sexual function, was analyzed by HPLC with photodiode array and mass spectral detection and found to contain a compound related to the synthetic phosphodiesterase-5 (PDE-5) inhibitors. Based on UV spectra, mass spectra and direct infusion $\mathrm{MS}^{n}$, the structure of the compound was tentatively identified as a sildenafil analogue in which the sulfonyl group had been replaced with an acetyl group. This new analogue is similar to acetildenafil, a previously reported sildenafil analogue, but differs in that it contains an $\mathrm{N}$-methyl group where acetildenafil contains an $\mathrm{N}$-ethyl group. The structure of the unknown was unequivocally established by chemical cleavage of the phenacylamine group of the molecule to generate $N$-methylpiperazine; other cleavage products matched those generated from acetildenafil. Since the new compound has one less $\mathrm{CH}_{2}$ group than acetildenafil, it was named nor-acetildenafil.
\end{abstract}

(C) 2007 Elsevier B.V. All rights reserved.

Keywords: Sildenafil analogue; Acetildenafil; Nor-acetildenafil; Erectile dysfunction; Phosphodiesterase-5 inhibitor; Dietary supplement; Liquid chromatography-mass spectrometry (LC-MS)

\section{Introduction}

The first drug approved for the treatment of erectile dysfunction (ED), sildenafil citrate, was introduced onto the market in 1998. Today, there are three phosphodiesterase-5 (PDE-5) enzyme inhibitors that have been approved by the U.S. Food and Drug Administration for the treatment of ED. They are sildenafil citrate (Viagra ${ }^{\circledR}$, manufactured by Pfizer), vardenafil hydrochloride (Levitra ${ }^{\circledR}$, manufactured by Bayer), and tadalafil (Cialis ${ }^{\circledR}$, manufactured by Lilly). With the commercial success of this new class of drugs, a number of over-the-counter herbal products have been marketed as natural alternatives to these synthetic prescription drugs. In recent years, there have been several reports of adulteration of such herbal dietary supplements with one of the three synthetic ED drugs [1-6] or an analogue in which minor modifications were made to the molecular structure of one of the ED drugs [5-16].

\footnotetext{
* Corresponding author. Tel.: +1 314539 3855; fax: +1 3145392113 . E-mail address: john.reepmeyer@fda.hhs.gov (J.C. Reepmeyer).
}

The first report [7] of an ED drug analogue as an illegal additive came with the detection of homosildenafil in a food beverage marketed for penile erectile dysfunction. The compound was isolated as a crystalline solid and characterized using UV spectroscopy, IR, high-resolution FAB-MS, and NMR. Another sildenafil analogue, acetildenafil, was detected in herbal products and characterized by several research groups [6,8-10]. Shin et al. [8] elucidated its structure using extensive NMR studies. Blok-Tip et al. [9] detected three sildenafil analogues, homosildenafil, acetildenafil, and hydroxyhomosildenafil, in herbal products and characterized their structures using LC-electrospray ionization (ESI)-MS, direct infusion ESI-MS ${ }^{n}$, UV, IR and NMR. Lai et al. [10] isolated acetildenafil from a dietary supplement and characterized the compound using 1D and 2D NMR spectroscopy, UV spectroscopy, and LC-MS-MS. A laboratory working with the Health Science Authority of Singapore detected sildenafil, vardenafil, hydroxyhomosildenafil, homosildenafil and acetildenafil in six pre-market dietary supplement bulk powders and one dietary supplement capsule [6]. This same research group detected hydroxyacetildenafil in a bulk powder dietary supplement and elucidated its structure using NMR, ESI-MS ${ }^{n}$ 


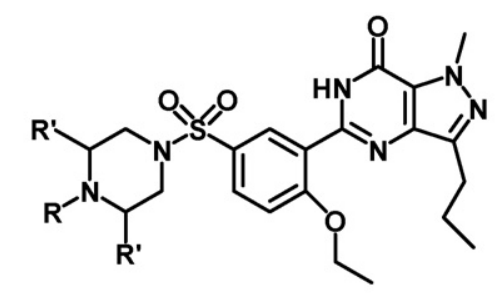

sildenafil (Viagra) $\mathrm{R}=\mathrm{CH}_{3} \mathrm{R}^{\prime}=\mathrm{H}$

homosildenafil $\mathrm{R}=\mathrm{CH}_{3} \mathrm{CH}_{2} \mathrm{R}^{\prime}=\mathrm{H}$

hydroxy-homosildenafil $\mathrm{R}=\mathrm{HOCH}_{2} \mathrm{CH}_{2} \mathrm{R}^{\prime}=\mathrm{H}$

methisosildenafil $\mathrm{R}=\mathrm{H} \quad \mathrm{R}^{\prime}=\mathrm{CH}_{3}$

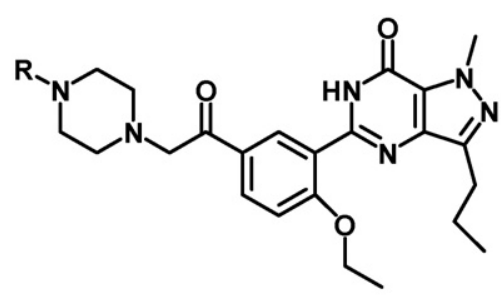

nor-acetildenafil $\mathrm{R}=\mathrm{CH}_{3}$

acetildenafil $\mathrm{R}=\mathrm{CH}_{3} \mathrm{CH}_{2}$

hydroxy-acetildenafil $\mathrm{R}=\mathrm{HOCH}_{2} \mathrm{CH}_{2}$

Fig. 1. Structures of sildenafil and some sildenafil analogues. Nor-acetildenafil is the new analogue reported in this paper.

and high resolution MS [11]. Fourier transform ion cyclotron resonance mass spectrometry (FTICRMS) was used to determine the accurate mass of several ED drug analogues and to study their fragmentation patterns. This accurate mass data was used to provide evidence for a new sildenafil analogue called piperidino-acetildenafil [12]. Recently, a sildenafil analogue, called methisosildenafil, was found to have methyl groups attached to carbon atoms rather than the nitrogen atom of the piperazine ring [13]. The structures of sildenafil and several of these sildenafil analogues are shown in Fig. 1.

Most of the analogues detected to date are analogues of sildenafil, probably because sildenafil was the first ED drug marketed. To our knowledge, only one tadalafil analogue and one vardenafil analogue have been detected as adulterants in herbal dietary supplements. Aminotadalafil was characterized using LC-UV, high-resolution MS, MS-MS, IR, and 2D NMR [14]. The UV spectrum of the compound was similar to tadalafil, and high resolution MS established a molecular formula indicating that the compound possessed one more nitrogen, one less carbon, and one less hydrogen atom than tadalafil. This data along with 2D NMR established the structure of aminotadalafil. The structure of piperidenafil, a vardenafil analogue, was elucidated by LC-UV-MS, direct infusion $\mathrm{MS}^{n}$, and hydrolysis of the sulfonamide bond followed by GC-MS and LC-MS analysis of the resulting amine and sulfonic acid products [15]. Aminotadalafil and piperidenafil have been further characterized by accurate mass determination using FTICRMS [12].

This paper reports on the detection and identification of a new sildenafil analogue found in an herbal dietary supplement. This analogue has an acetyl group in place of the sulfonyl group of sildenafil, and differs from acetildenafil in that it has an $\mathrm{N}$ methylpiperazine where acetildenafil has an $\mathrm{N}$-ethylpiperazine. Since the new compound has one less $\mathrm{CH}_{2}$ group than acetildenafil, it was named nor-acetildenafil. Its structure is given in Fig. 1. A technique that proved useful in the structure elucidation of analogues of vardenafil [15] and sildenafil [13] was the hydrolytic cleavage of the sulfonamide bond, followed by individual characterization of the cleavage products. It allowed for the determination of the amine portion of the molecule using GC-MS aided with a spectral library search, and allowed one to compare cleavage products of the analogue to those of the par- ent drug. Since the new analogue has no sulfonamide bond, an alternative reaction was developed to cleave the phenacylamine $\mathrm{C}-\mathrm{N}$ bond.

\section{Experimental}

\subsection{Chemicals}

Sildenafil citrate reference standard was obtained from Pfizer, tadalafil reference standard from Lilly, and vardenafil hydrochloride trihydrate reference standard from Bayer. 1Methylpiperazine and anhydrous sodium sulfate $\left(\mathrm{Na}_{2} \mathrm{SO}_{4}\right.$, ACS reagent) were purchased from Aldrich (Milwaukee, WI, USA), dimethyl sulfoxide (DMSO) ACS reagent from Sigma (St. Louis, MO, USA), sodium hydroxide ( $\mathrm{NaOH}$, molecular biology grade) from Fisher Scientific (Fair Lawn, NJ, USA), sodium acetate anhydrous $(\mathrm{NaOAc})$ from Taylor Chemical (St. Louis, MO, USA) and benzoyl chloride (analytical reagent) from Mallinckrodt (St. Louis, MO, USA). Formic acid (reagent grade, min 98\%) and HPLC-grade Omni-Solve acetonitrile (MeCN), methanol $(\mathrm{MeOH})$ and ethyl acetate $(\mathrm{EtOAc})$ were purchased from EM Science (Gibbstown, NJ, USA). Water was purified using a Milli-Q Water System to $18 \mathrm{M} \Omega \mathrm{cm}$ (Millipore, Bedford, MA, USA).

\subsection{Standard and sample preparation}

A working standard solution of vardenafil hydrochloride, sildenafil citrate, and tadalafil was prepared in $\mathrm{MeCN}-\mathrm{H}_{2} \mathrm{O}(1: 1)$ with each compound at a concentration of $\sim 25 \mu \mathrm{g} \mathrm{ml}^{-1}$ for analysis by LC-MS. Two herbal dietary supplements with the same name and the same distributor, one packaged as a single capsule in a blister pack (product 1 ) and the other as 12 capsules in a bottle (product 2), were purchased over the Internet. Two different composites were prepared, one for each of the products. The contents from two capsules of product 1 were mixed and vortexed briefly to provide a uniform mixture. The same procedure was applied to three capsules of product 2 . Approximately $30 \mathrm{mg}$ of each composite was extracted with $5 \mathrm{ml} \mathrm{MeOH}$ in a glass tube sealed with a PTFE-lined cap. The mixture was vortexed vigorously for $30 \mathrm{~s}$, placed in a ultrasonic bath for $10 \mathrm{~min}$, 
the process repeated, vortexed one more time, then centrifuged for $15 \mathrm{~min}$ (Centrifig, model 228, bench-top centrifuge $3300 \mathrm{rpm}$ maximum speed, Fisher Scientific, Pittsburgh, PA, USA). The supernatant liquid was decanted to a second test tube and stored in a refrigerator for subsequent analyses. A 50- $\mu$ l portion of the methanol sample extract was diluted to $2 \mathrm{ml}$ with $\mathrm{MeCN}-\mathrm{H}_{2} \mathrm{O}$ $(1: 1)$ and the solution was analyzed by LC-MS.

\section{3. $L C-M S$}

The samples were screened for ED drugs or analogues based on an LC-MS method previously described [5,13]. The LC-MS system consisted of an Agilent 1100 HPLC system with a binary

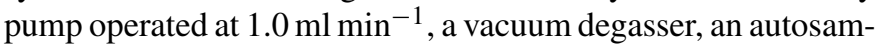
pler set to inject $10 \mu \mathrm{l}$, a column oven thermostatted at $30^{\circ} \mathrm{C}$, a diode array detector monitored at $230 \mathrm{~nm}$ and set to acquire spectra of peaks detected, and an Agilent quadrapole mass spectrometer, model G1946D, controlled with ChemStation software, version B.02.01. The mass spectrometer was operated in the positive ion mode with electrospray ionization; the scan range was 130-700 amu. The stationary phase consisted of Zorbax stable bond C-18 packing, $150 \mathrm{~mm} \times 4.6 \mathrm{~mm}, 5 \mu \mathrm{m}$ particle size (Agilent Technologies, Wilmington, DE, USA), with a BrownLee NewGuard C-18 guard column, $15 \mathrm{~mm} \times 3.2 \mathrm{~mm}$, $7 \mu \mathrm{m}$ particle size (Applied Biosystems, Foster City, CA, USA). Two mobile solvents consisted of $0.1 \%$ formic acid in water (A) and $0.1 \%$ formic acid in $\mathrm{MeCN}(\mathrm{B})$. The mobile phase consisted of $15 \% \mathrm{~B}$ for the first $3 \mathrm{~min}$, changed linearly to $100 \% \mathrm{~B}$ over $3-15$ min, and held at $100 \% \mathrm{~B}$ until the end of the 20 min analysis time.

\subsection{Direct infusion $M S^{n}$}

A $20 \mu$ l portion of the LC-MS sample solution (Section 2.2) of the herbal product was mixed with $200 \mu \mathrm{l}$ of $0.1 \%$ formic acid in $\mathrm{MeCN}-\mathrm{H}_{2} \mathrm{O}(1: 1)$ and analyzed by direct infusion at $3 \mu \mathrm{l} \mathrm{min}{ }^{-1}$ into a Thermo-Finnigan LCQ XP ion trap mass spectrometer. The instrument was operated in electrospray positive ion mode with the following parameters: sheath gas 5 (arbitrary units), auxiliary gas 0 (arbitrary units), spray voltage $5 \mathrm{kV}$, capillary voltage $7 \mathrm{~V}$, ion transfer capillary temperature $250^{\circ} \mathrm{C}$. Other parameters were set during instrument calibration. Collision induced dissociation (CID) MS was also applied to acetildenafil for comparison to the unknown.

\subsection{Chemical cleavage of the unknown}

The molecule of the unknown (referred to as compound X) was cleaved in order to identify the amine portion of the molecule and to compare the other cleavage products to the cleavage products from acetildenafil. Two $100-\mu 1$ portions of the $\mathrm{MeOH}$ herbal extract were evaporated to dryness in separate 1-ml ReactiVials $^{\mathrm{TM}}$ (Pierce, Rockford, IL, USA). The residue in one vial was dissolved in $100 \mu \mathrm{l}$ of DMSO and $4 \mathrm{mg}$ anhydrous $\mathrm{NaOAc}$ was added; the $\mathrm{NaOAc}$ catalyst does not dissolve. The mixture was heated at $60^{\circ} \mathrm{C}$ for $4 \mathrm{~h}$ in a Reacti-Therm ${ }^{\mathrm{TM}}$ heating module (Pierce, Rockford, IL, USA). A methanol solution of acetilde- nafil was evaporated and the residue treated in a similar manner. The residue of compound $\mathrm{X}$ in the second vial was dissolved in $100 \mu \mathrm{l}$ of $\mathrm{MeOH}$, mixed with $50 \mu \mathrm{l}$ of $3 \mathrm{M} \mathrm{NaOH}$, and heated at $95^{\circ} \mathrm{C}$ for $3 \mathrm{~h}$.

\subsubsection{LC-MS analysis of the cleavage reaction solution}

Portions $(20-\mu \mathrm{l})$ of each DMSO reaction solution were diluted separately with $1 \mathrm{ml} \mathrm{MeCN}-\mathrm{H}_{2} \mathrm{O}(1: 1)$ and analyzed by LC-MS.

\subsection{2. $G C-M S$ analysis of the amine cleavage product}

GC-MS was used to detect and identify the free amine compound generated by cleavage of compound $\mathrm{X}$ in $\mathrm{MeOH}$ with $\mathrm{NaOH}$; the $\mathrm{MeOH}$ reaction solution was used rather than the DMSO reaction solution to avoid the use of the high boiling solvent on the GC column. Analyses were conducted on a Hewlett-Packard gas chromatograph, model 5890 series II, with a Hewlett-Packard 5972 mass selective detector using a DB-5MS, $30 \mathrm{~m} \times 0.25 \mathrm{~mm}$ capillary column, $0.5 \mu \mathrm{m}$ film thickness, under the following conditions-injector: $63{ }^{\circ} \mathrm{C}$ at start for on-column injection and automatically increased to a set point of $220^{\circ} \mathrm{C}, 0.5 \mu \mathrm{l}$ injection volume on-column; carrier gas: helium at $0.8 \mathrm{ml} \mathrm{min}^{-1}$ constant flow; oven temperature: $60{ }^{\circ} \mathrm{C}$ for $3 \mathrm{~min}$, increased to $220^{\circ} \mathrm{C}$ at $20^{\circ} \mathrm{C} \mathrm{min}{ }^{-1}$; MS scan range 25-150 amu. A standard solution was prepared containing $N$ methylpiperazine at a concentration of $1 \mathrm{mg} \mathrm{ml}^{-1}$ in methanol and analyzed in the same manner.

\subsubsection{LC-MS analysis of the benzoyl derivative of the amine cleavage product}

The benzoyl derivative of $N$-methylpiperazine standard was prepared via the Schotten-Baumann reaction, a classic organic reaction used for formation of an amide from an amine and an acid chloride in a dilute aqueous alkaline solution. Thus, a mixture of $10 \mu \mathrm{l} N$-methylpiperazine, $1 \mathrm{ml}$ water, $30 \mu \mathrm{l}$ benzoyl chloride $(\sim 0.35 \mathrm{mmol})$, and $300 \mu \mathrm{l}$ of $2 \mathrm{M} \mathrm{NaOH}$ ( $1 \mathrm{mmol}$ ) was stirred. Additional $100-\mu$ l portions of $2 \mathrm{M} \mathrm{NaOH}$ were added, if necessary, to ensure that the solution was strongly basic to $\mathrm{pH}$ paper. The mixture was stirred for $3 \mathrm{~min}$, then an additional $30 \mu$ l benzoyl chloride and $300 \mu \mathrm{l}$ of $2 \mathrm{M} \mathrm{NaOH}$ were added. The addition of benzoyl chloride and $2 \mathrm{M} \mathrm{NaOH}$ was continued every 3 min until a total of six additions were made, always ensuring that the mixture remained basic. After the last addition, the mixture was stirred for an additional $2 \mathrm{~h}$, with the addition of $2 \mathrm{M} \mathrm{NaOH}$, if necessary, to maintain a basic solution. The aqueous solution was extracted three times with $1 \mathrm{ml}$ EtOAc. The EtOAc extracts were combined, dried over anhydrous $\mathrm{Na}_{2} \mathrm{SO}_{4}$, decanted to a second tube, and back extracted into $1.5 \mathrm{ml}$ of $0.1 \%$ formic acid in water. The aqueous formic acid solution was analyzed by LC-DAD-MS using the method described in Section 2.3.

The amine generated by cleavage of compound $\mathrm{X}$ was derivatized with benzoyl chloride in the same manner by first adding $500 \mu \mathrm{l}$ water to the $\mathrm{NaOH} / \mathrm{MeOH}$ reaction solution, transferring the solution to a test tube, and proceeding as described for the $N$-methylpiperazine standard. 

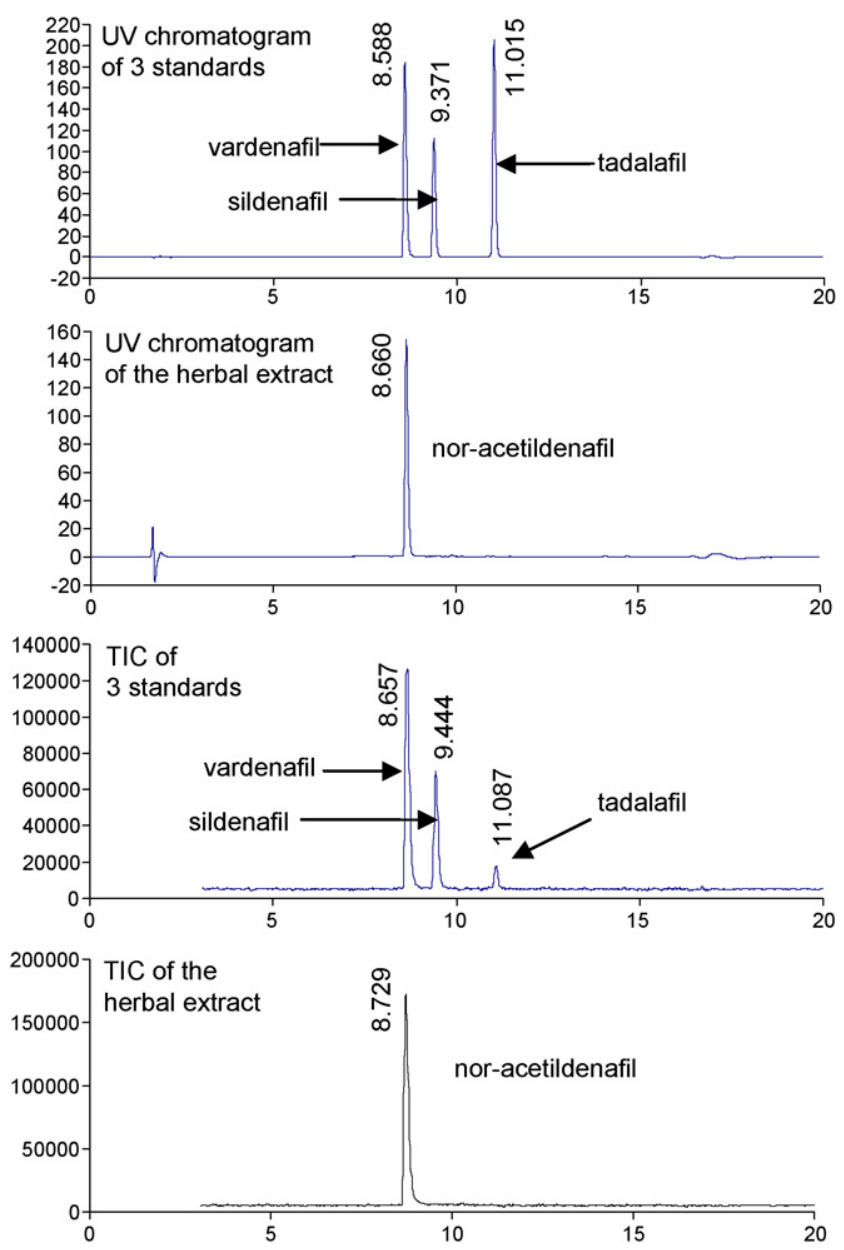

Fig. 2. UV chromatograms and total ion chromatograms (TIC) of a mixture of sildenafil, vardenafil and tadalafil standards, and the adulterated herbal drug.

\section{Results and discussion}

\section{1. $L C-D A D-M S$}

One prominent peak was seen in the UV chromatogram and total ion chromatogram of the methanol extract of the herbal dietary supplement (Fig. 2). This component (compound X) had a retention time almost the same as that of vardenafil. However, both the UV and mass spectra of vardenafil differ from those of compound $\mathrm{X}$. The mass spectrum of compound $\mathrm{X}$ has a protonated molecule $[M+\mathrm{H}]^{+}$at $m / z, 453$ and a doubly charged species $[M+2 \mathrm{H}]^{2+}$ at $m / z 227$ (Fig. 3a). The UV spectrum showed two distinct $\lambda_{\max }$ at 234 and $280 \mathrm{~nm}$, with the longer wavelength peak slightly more intense (Fig. 3b). While the UV spectrum of compound $\mathrm{X}$ differs considerably from the spectra of the three ED drugs, each of which differ from one another, it appears to correspond to the reported $\lambda_{\max }$ of 234 and $279 \mathrm{~nm}$ [9] and published UV spectra $[6,10]$ of acetildenafil, a sildenafil analogue that has been previously characterized. A sample of acetildenafil, which had been isolated previously from an herbal dietary supplement in our laboratory and characterized by comparison to literature data [9], was used for comparison to compound $\mathrm{X}$ by LC-DAD-MS. The UV spectrum of compound $\mathrm{X}$ and
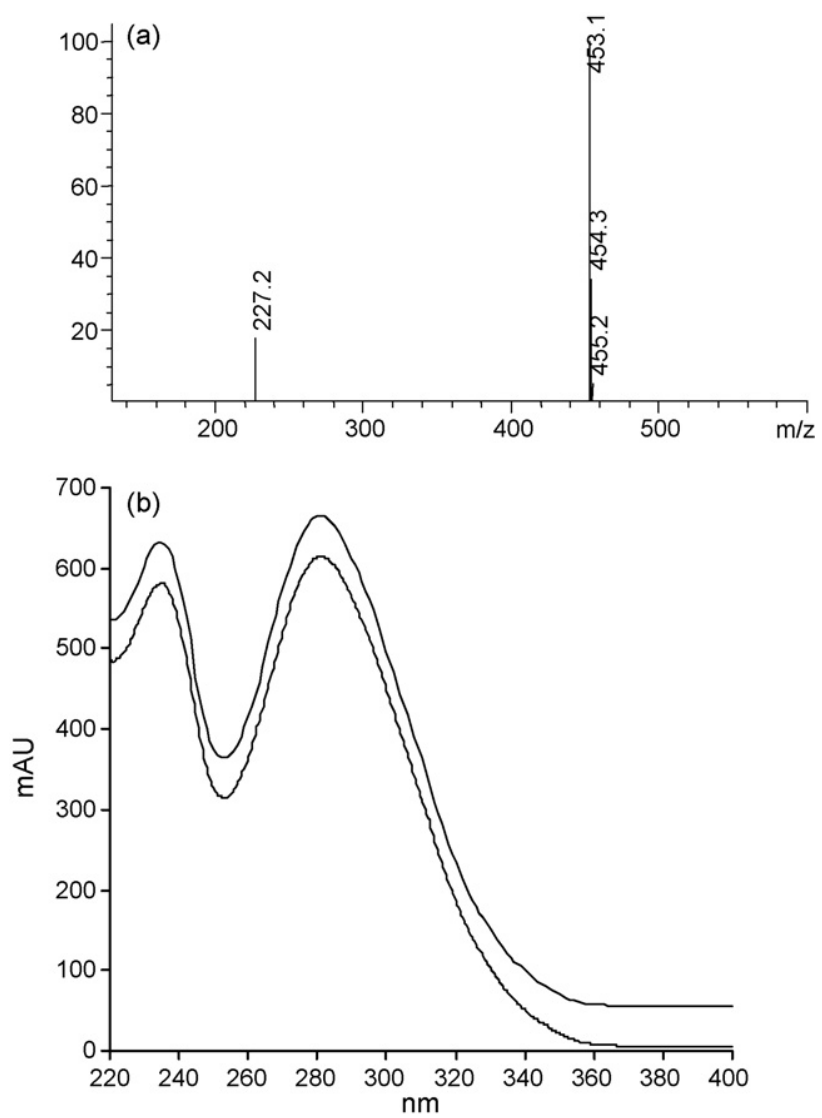

Fig. 3. (a) Mass spectrum of the herbal component (nor-acetildenafil). (b) UV spectra of acetildenafil (lower trace) and nor-acetildenafil (upper trace); spectra are normalized and offset.

acetildenafil are identical (Fig. 3b), and thus, compound $\mathrm{X}$ and acetildenfil probably have the same chromophore. This preliminary evidence suggests that compound X, like acetildenafil, possesses an acetyl group attached to the benzene ring instead of a sulfonyl group. Further support for this structural feature is shown by mass spectral data and a chemical cleavage reaction which are discussed below. Furthermore, acetildenafil had a retention time slightly longer than compound $\mathrm{X}$ and a protonated molecule at $m / z 467,14$ units above that of compound $X$, strongly suggesting that compound $\mathrm{X}$ has one less $\mathrm{CH}_{2}$ group in the molecule.

\subsection{Direct infusion $M S^{n}$}

The mass spectrum resulting from collision induced dissociation of the protonated molecule of acetildenafil is shown in Fig. 4(a). A collision energy of 40 was selected in order to duplicate the fragmentation pattern reported in the literature, and the spectrum does correlate well with the published spectrum [9]. The CID-MS on the protonated molecule of compound $\mathrm{X}$ (nor-acetildenafil), also at a collision energy of 40, is shown in Fig. 4(b). Acetildenafil and compound $\mathrm{X}$ have common mass losses of 18, 28, 43 and 47 and common product ions at $\mathrm{m} / \mathrm{z}$ 396, 355, 353, 341, 339 and 325. A proposed fragmentation scheme is presented in Fig. 5. Both compound $\mathrm{X}$ and acetildenafil fragment across the piperazine ring (Fig. 5, fragmentation 


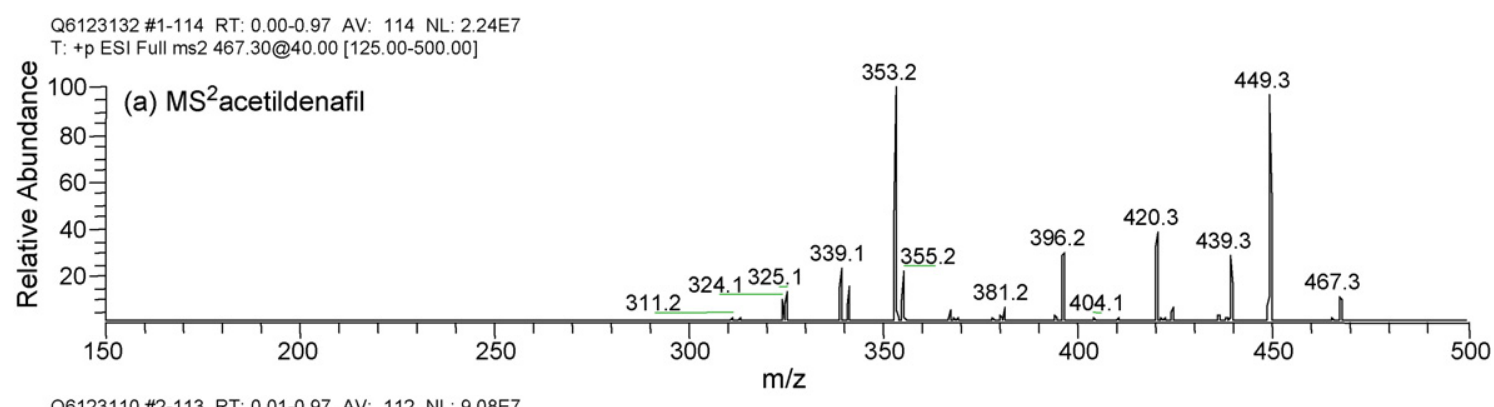

Q6123110 \#2-113 RT: 0 01-0.97 AV: 112 NL: $9.08 E 7$

T: +p ESI Full ms2 453.30@40.00 [125.00-500.00]

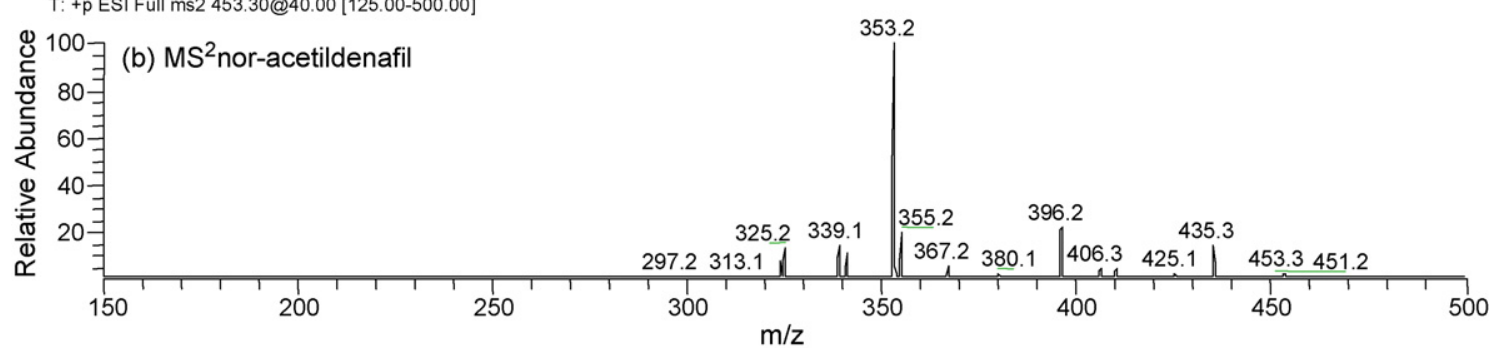

Q6123136 \#1-91 RT: 0.00-0.97 AV: 91 NL: 8.90E6

T: +p ESI Full ms3467.30@40.00353.20@40.00 [95.00-500.00]

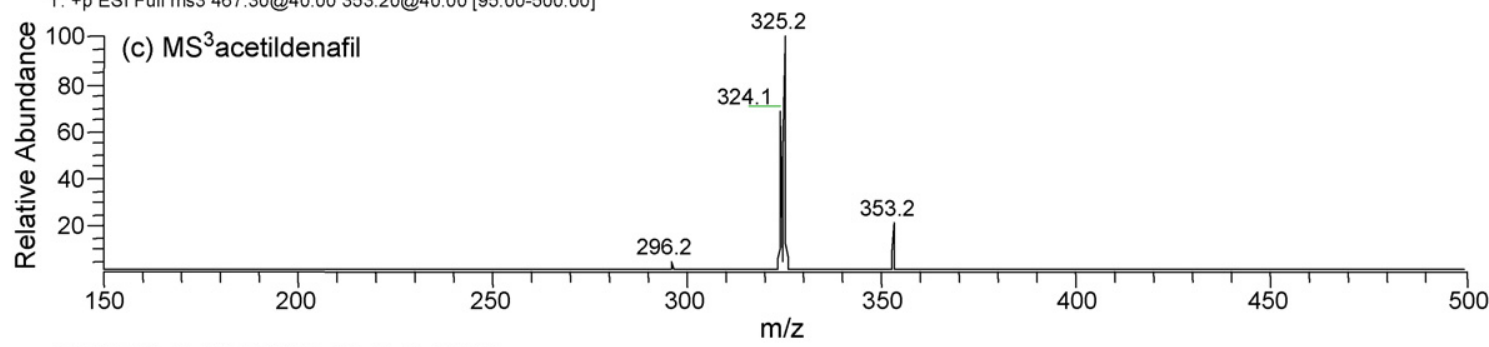

Q6123125 \#1-94 RT: 0.00-1.00 AV: 94 NL: 3.27E7

T: +p ESI Full ms3453.30@40.00353.20@40.00 [95.00-500.00]

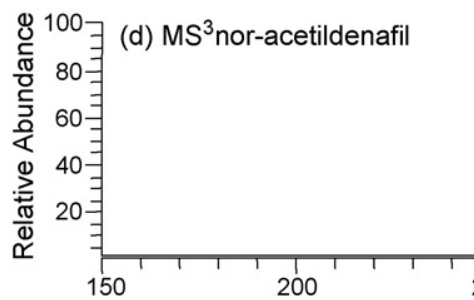

(d) $\mathrm{MS}^{3}$ nor-acetildenafi

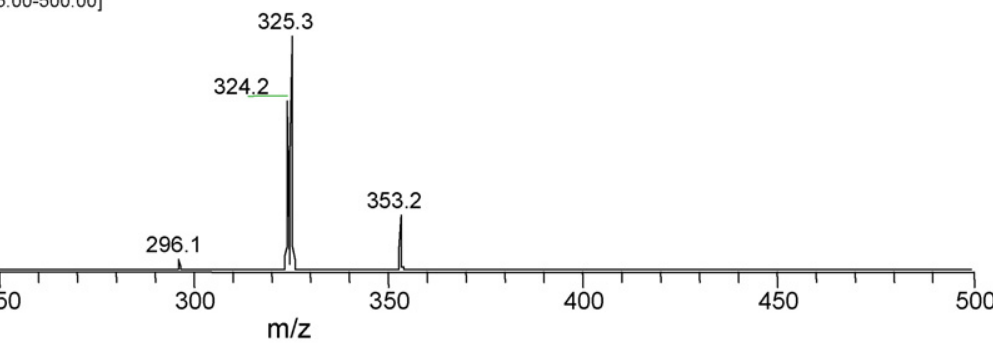

Fig. 4. CID mass spectra generated from (a) the protonated molecule $(\mathrm{m} / \mathrm{z}, 467)$ of acetildenafil, (b) the protonated molecule $(\mathrm{m} / \mathrm{z}, 453)$ of nor-acetildenafil, (c) the prominent product ion at $\mathrm{m} / \mathrm{z}, 353$ of acetildenafil, and (d) the prominent product ion at $\mathrm{m} / \mathrm{z}, 353$ of nor-acetildenafil. Matching spectra shown in (c) and (d) support the contention that the product ions $\mathrm{m} / z 353$ from acetildenafil and nor-acetildenafil are the same.

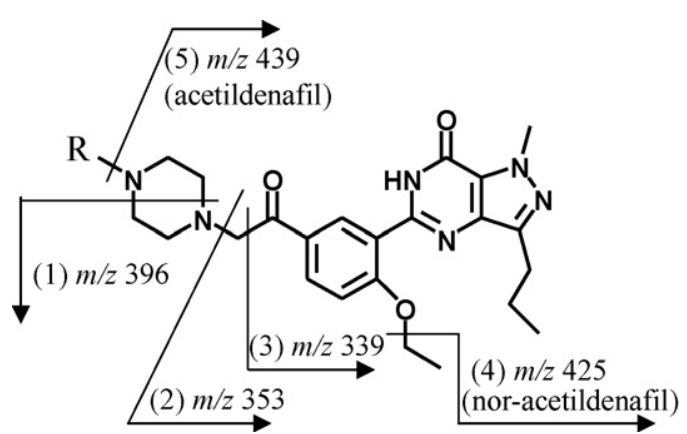

Fig. 5. Proposed fragmentation of the protonated molecules of acetildenafil $\left(\mathrm{R}=\mathrm{CH}_{3} \mathrm{CH}_{2}-,[M+\mathrm{H}]^{+} m / z 467\right)$ and nor-acetildenafil $\left(\mathrm{R}=\mathrm{CH}_{3}-,[M+\mathrm{H}]^{+}\right.$ $m / z, 453)$.
1) to give $m / z 396$ with losses of $57\left(-\mathrm{C}_{3} \mathrm{H}_{7} \mathrm{~N}\right)$ and $71\left(-\mathrm{C}_{4} \mathrm{H}_{9} \mathrm{~N}\right)$, respectively. This is the highest mass fragment common to both compounds. Acetildenafil and compound X fragment with mass losses of 114 and 100, respectively, to generate a major product ion at $m / z$ 353. This loss in acetildenafil is attributed to a neutral loss of $N$-ethylpiperazine, and an analogous loss in compound $\mathrm{X}$ would be a neutral loss of methylpiperazine (Fig. 5, fragmentation 2). The prominent ion at 353 , common to both compounds, provides additional evidence that the two compounds differ by a $\mathrm{CH}_{2}$ group, and furthermore, that this $\mathrm{CH}_{2}$ is associated with the piperazine ring of the molecule. If the product ion at $\mathrm{m} / \mathrm{z}, 353$ is the same for both acetildenafil and compound $\mathrm{X}$, then further fragmentation of this ion should be the same for both compounds. Fragmentation of product ion $\mathrm{m} / \mathrm{z}$ 353 for acetildenafil and compound X, shown in Fig. 4(c) and (d), 
are practically identical, generating two prominent product ions at $m / z 325,324$ and a minor ion at 296. The fragments at $m / z, 325$ and 324 are attributed to fragmentation 2 followed by losses of $\mathrm{CH}_{2}=\mathrm{CH}_{2}$ and $\mathrm{CH}_{3} \mathrm{CH}_{2}$, respectively, through fragmentation 4. Fragmentation 3 (Fig. 5) generates the acylium ion at $m / z 339$. Further fragmentation of both compounds $\left(\mathrm{ms}^{4}\right)$ gives a common transition of $[M+\mathrm{H}]^{+} \rightarrow 353 \rightarrow 325 \rightarrow 308,297(100 \%)$, and 281 at approximately the same relative abundance (data not shown). The UV and mass spectra, relative retention times, and mass fragmentation patterns establish that the structures of compound $\mathrm{X}$ and acetildenafil differ by a $\mathrm{CH}_{2}$ group in the amine moiety of the molecule, and is consistent for a structure in which the $N$-ethylpiperazine of acetildenafil is replaced with a methylpiperazine. However, one cannot assume that the methyl group is attached to the nitrogen atom. Indeed, one sildenafil analogue, recently detected as an adulterant in a herbal dietary supplement, was found to have methyl groups attached to carbon atoms of a piperazine ring [13].

\subsection{Chemical cleavage of compound $X$}

In the structure elucidation of analogues of sildenafil [13] and vardenafil [15], a hydrolytic technique was used to cleave the sulfonamide bond, thus releasing the free amine. This amine was subsequently identified by GC-MS and confirmed by LC-MS of a benzoyl derivative of the amine. The suspect compound currently under investigation has no sulfonamide group. However, it does have a phenacylamine group, and if the $\mathrm{C}-\mathrm{N}$ bond of this group were cleaved, it would generate the free amine, which could then be identified by GC-MS analysis.

Although there are several $\mathrm{C}-\mathrm{N}$ bonds in the molecule of acetildenafil and nor-acetildenafil, the $\mathrm{C}-\mathrm{N}$ bond of phenacylamines are more reactive than other $\mathrm{C}-\mathrm{N}$ bonds. The cleavage reaction developed here takes advantage of this selectivity. Numerous reactions with variations in the solvent and reagent were tried to establish a suitable cleavage process. Acidic conditions normally gave poor results, while neutral or basic conditions gave good results. The use of NaOAc in DMSO was sufficiently mild to give consistent results with the generation of two main products. The cleavage reaction was poor without the addition of NaOAc (e.g. DMSO alone). Methanol was more suitable than the high boiling DMSO as the solvent in the reaction intended for GC-MS analysis.

\subsection{1. $G C-M S$}

GC-MS analysis of the cleavage reaction solution showed a peak which had a mass spectrum that gave a top match for $N$-methylpiperazine in the NIST mass spectral library. Concomitant injection of $\mathrm{N}$-methylpiperazine standard and the amine generated from compound $\mathrm{X}$ into the GC-MS gave retention times of 4.29 and 4.17 min, respectively, and matching mass spectra with molecular ions at $\mathrm{m} / \mathrm{z} 100$ (13\% relative abundance), and prominent ions at $m / z 58$ (100\% relative abundance), 43, 42, and 28 .

\subsubsection{LC-MS of benzoyl derivative of the amine product}

The cleavage reaction solution was treated with benzoyl chloride under Schotten-Baumann conditions to form a benzamide. When analyzed by LC-MS, the benzoyl derivatives of the amine formed from compound $\mathrm{X}$ and the benzoyl derivative of $\mathrm{N}$ methylpiperazine standard gave retention times of 3.74 and $3.69 \mathrm{~min}$, respectively. Both compounds had ions at $m / z 205$ $\left([M+\mathrm{H}]^{+}, 100 \%\right.$ relative abundance $)$ and $m / z 409\left(\left[M_{2}+\mathrm{H}\right]^{+}\right.$, $\sim 40 \%$ relative abundance, a dimer). Although there was no distinct $\lambda_{\max }$ in the UV spectra of the two compounds, their spectra were identical. Data are consistent with the GC-MS data and establish the structure of $N$-methylpiperazine.

\subsubsection{LC-MS analysis of cleavage reaction products}

The cleavage of compound $\mathrm{X}$ establishes that $\mathrm{N}$-methylpiperazine is part of its structure. If both acetildenafil and noracetildenafil are cleaved and the piperazine ring is lost, then the cleavage product should be the same for both compounds, assuming the assigned structure for compound $\mathrm{X}$ is correct. In
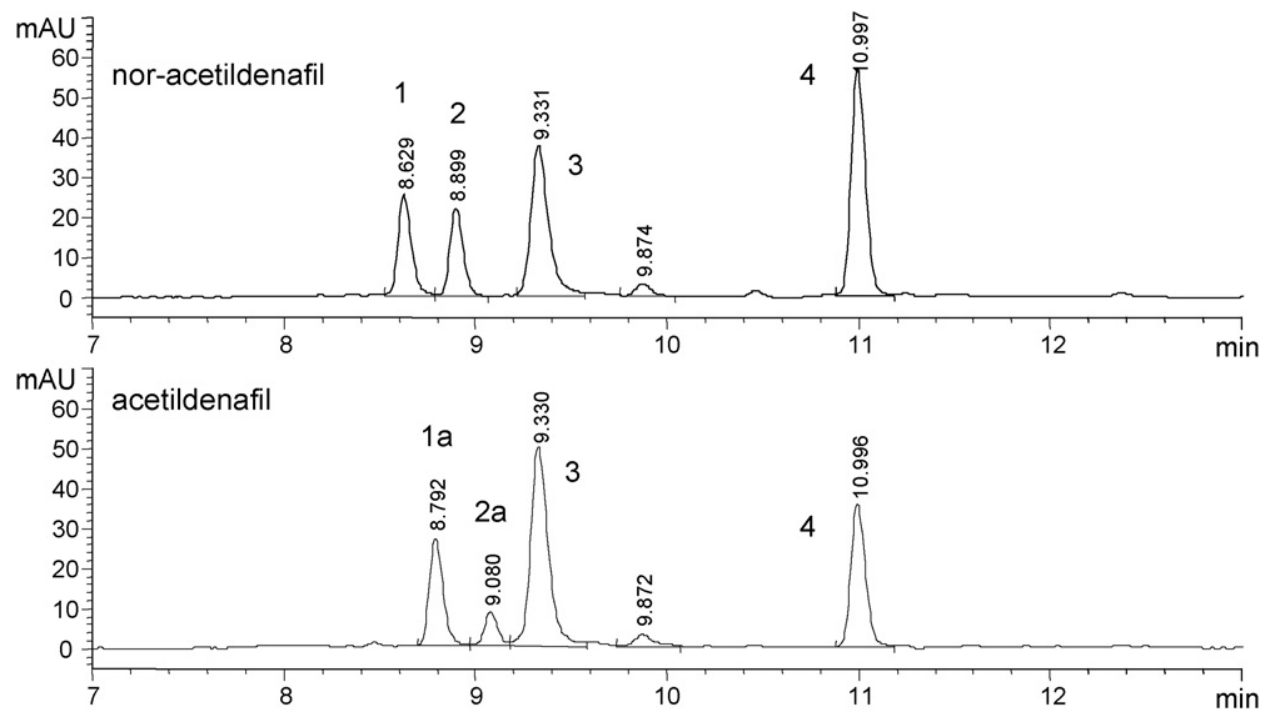

Fig. 6. UV chromatograms of reaction products of acetildenafil and nor-acetildenafil when treated with NaOAc in DMSO at $60^{\circ} \mathrm{C}$ for $4 \mathrm{~h}$. 
contrast, if the piperazine ring remains intact, then the acetildenafil cleavage product will have one $\mathrm{CH}_{2}$ group more than the nor-acetildenafil cleavage product. Since the phenacylamine $\mathrm{C}-\mathrm{N}$ bond is the target for cleavage and it has been shown in Sections 3.3.1 and 3.3.2 that $N$-methylpiperazine is generated from compound $\mathrm{X}$, then one would expect to see at least one cleavage product common to acetildenafil and compound X. Fig. 6 shows the UV chromatograms of the two reaction solutions when compound $X$ (nor-acetildenafil) and acetildenafil were treated with $\mathrm{NaOAc}$ in DMSO. Peaks 1 and $1 \mathrm{a}$ are the starting materials, noracetildenafil and acetildenafil. Peak 4 from compound $X$ has the same retention time, UV spectrum, and mass spectrum $(\mathrm{m} / \mathrm{z} 357)$ as peak 4 from acetildenafil. Similarly, peak $3(\mathrm{~m} / \mathrm{z} 385)$ is the same for both compounds. The proposed structures for 3 and 4 are the aryl glyoxylic acid and aryl carboxylic acid products, with 3 derived from the cleavage of the phenacyl $\mathrm{C}-\mathrm{N}$ bond and 4 derived from 3 . As the reaction continues, peak 4 continues to grow, while the other peaks decline. The proposed scheme for the cleavage reaction in DMSO is as follows:

$$
\begin{aligned}
& \mathrm{Ar}-\mathrm{CO}-\mathrm{CH}_{2}-\mathrm{NR}_{2} \\
& \quad \rightarrow \mathrm{Ar}-\mathrm{CO}-\mathrm{COOH}+\mathrm{Ar}-\mathrm{COOH}+\mathrm{HNR}_{2}
\end{aligned}
$$

where Ar represents the substituted benzene ring of noracetildenafil or acetildenafil and $\mathrm{NR}_{2}$ represents the piperazine ring.

Peak $2(\mathrm{~m} / \mathrm{z} 467)$ from nor-acetildenafil corresponds to peak $2 \mathrm{a}(\mathrm{m} / \mathrm{z} 481)$ from acetildenafil; the structure of these compounds are unknown, but since the compounds differ in mass by 14 units, these compounds are not a result of phenacylamine cleavage and the piperazine ring is still attached. The fact that compound $\mathrm{X}$ and acetildenafil have common cleavage products is additional proof for the structure of nor-acetildenafil.

\section{Conclusion}

A product, marketed as a natural herbal dietary supplement and advertised as a sexual performance enhancer for men, was found to contain an analogue of an erectile dysfunction drug. This compound, named nor-acetildenafil, may be described as an analogue of sildenafil in which the sulfonyl group has been replaced with an acetyl group and as an analogue of acetildenafil in which the $N$-ethyl group on piperazine has been replaced with an $N$-methyl group. The structure of the compound was elucidated using LC-UV-ESI-MS, direct infusion $\mathrm{MS}^{n}$, and chemical cleavage of the molecule followed by GC-MS and LC-MS analysis of the cleaved products. Two lots of the herbal product were found to contain 79 and $85 \mathrm{mg}$ per capsule as the free base when analyzed against sildenafil citrate reference standard. Viagra is available in doses of 25,50 , and $100 \mathrm{mg}$.

The adulteration of "natural" herbal dietary supplements with erectile dysfunction drugs or analogues is a growing trend and posses a health threat to patients who unwittingly consume a synthetic drug that has been untested for safety and efficacy.

\section{Acknowledgement}

The authors thank Benjamin Westenberger for initiating this study.

\section{References}

[1] X. Zhu, S. Xiao, B. Chen, F. Zhang, S. Yao, Z. Wan, D. Yang, H. Han, J. Chromatogr. A 1066 (2005) 89-95.

[2] A.J. Sabucedo, M.A. Gutierrez, K.C. Mueller, B.L. Bellissima, Y.L. Hsu, S. Rose, K.G. Furton, J. Am. Med. Assoc. 291 (2004) 560-562.

[3] E. Mikami, T. Ohno, H. Matsumoto, Forensic Sci. Int. 130 (2002) 140146.

[4] N. Fleshner, M. Harvey, H. Adomat, C. Wood, A. Eberding, K. Hersey, E. Guns, J. Urol. 174 (2005) 636-641.

[5] S.R. Gratz, C.L. Flurer, K.A. Wolnik, J. Pharm. Biomed. Anal. 36 (2004) $525-533$.

[6] P. Zou, S.S.-Y. Oh, P. Hou, M.-Y. Low, H.-L. Koh, J. Chromatogr. A 1104 (2006) 113-122.

[7] M.H. Shin, M.-K. Hong, W.S. Kim, Y.J. Lee, Y.C. Jeoung, Food Addit. Contam. 20 (2003) 793-796.

[8] C. Shin, M. Hong, D. Kim, Y. Lim, Magn. Reson. Chem. 42 (2004) 1060-1062.

[9] L. Blok-Tip, B. Zomer, F. Bakker, K.D. Hartog, M. Hamzink, J.T. Hove, M. Vredenbregt, D. de Kaste, Food Addit. Contam. 21 (2004) 737-748.

[10] K.-C. Lai, Y.-C. Liu, M.-C. Tseng, J.-H. Lin, J. Food Drug Anal. 14 (2006) 19-23.

[11] P. Hou, P. Zou, M.-Y. Low, E. Chan, H.-L. Koh, Food Addit. Contam. 23 (2006) 870-875

[12] S.R. Gratz, B.M. Gamble, R.A. Flurer, Rapid Commun. Mass Spectrom. 20 (2006) 2317-2327.

[13] J.C. Reepmeyer, J.T. Woodruff, D. André d'Avignon, J. Pharmaceut. Biomed. Anal. 43 (2007) 1615-1621.

[14] P. Zou, P. Hou, M.-Y. Low, H.-L. Koh, Food Addit. Contam. 23 (2006) 446-451.

[15] J.C. Reepmeyer, J.T. Woodruff, J. Chromatogr. A 1125 (2006) 67-75.

[16] S.S.-Y. Oh, P. Zou, M.-Y. Low, H.-L. Koh, J. Toxicol. Environ. Health A 69 (2006) 1951-1958. 